\title{
LA CONTROVERSIA SOBRE LA VIVISECCIÓN
}

\author{
Jorge Alberto Álvarez-Díaz*
}

Resumen: Este artículo revisa la vivisección en animales no humanos y su actual justificación desde el punto de vista bioético. Se inicia con una breve revisión histórica sobre la vivisección animal hasta el recrudecimiento de la polémica sobre ésta en el siglo XX. Reflexiona sobre la ética de la relación entre seres humanos y animales y de la experimentación con animales, y concluye que hoy podría ser justificable una vivisección animal sólo en situaciones verdaderamente excepcionales, de lo contrario no se estaría obrando conforme a la ética.

Palabras clave: experimentación con animales de laboratorio, vivisección, bienestar animal

\section{THE CONTROVERSY OVER VIVISECTION}

Abstract: The paper reviews the vivisection in nonhuman animals and whether it is justifiable nowadays from a bioethical point of view. The work departs with a brief historical perspective about the animal vivisection topic until the new outbreak of the controversy in the twentieth century. It reflects on the ethics of the relation between human beings and animals, concluding that nowadays animal vivisection could only be justified in truly exceptional situations; otherwise, it would be contrary to ethics.

Key words: laboratory animal experimentation, vivisection, animal welfare

\section{A CONTROVERSIA SOBRE A VIVISSECÇÃO}

Resumo: Este artigo faz uma revisão sobre a vivisseção em seres humanos em sua atual justificativa, a partir do ponto de vista bioético. Inicia com uma breve revisão histórica sobre a vivissecção animal, até o recrudescimento da polêmica sobre esta questão no século XX. Reflete sobre a ética da relação entre seres humanos e animais e da experimentação com animais. Conclui que hoje se poderia justificar uma vivissecção animal, somente em situações consideradas verdadeiramente excepcionais, do contrário não se estaria agindo eticamente.

Palavras chave: experimentação de animais em laboratório, vivissecção, bem-estar animal

* Médico, Sexólogo Clínico, Magíster en Bioética, México Correspondencia: bioetica_reproductiva@hotmail.com 


\section{Introducción}

El término "vivisección" deriva del latín vivus, vivo, y sectio-õnis, corte, esto es, la realización de cortes o disecciones en un organismo vivo. Se ha practicado - con defensores y detractores-en distintos períodos de la historia, tanto en animales humanos como no humanos ${ }^{1}$. En la Antigüedad se realizaron vivisecciones en condenados a muerte o en presos; hoy está claro que esta práctica no es éticamente aceptable. El desarrollo de este trabajo se refiere a la controversia sobre la vivisección en animales.

El uso de animales con el fin de adquirir conocimientos data de los inicios mismos de la historia. Aristóteles (384-322 a.C.), gran naturalista y filósofo, fue de los primeros en realizar disecciones (no vivisecciones) en animales, mostrando diferencias entre las especies. Otros realizaron vivisecciones posteriormente, pero, al mismo tiempo, aparecieron detractores a dicha práctica. Celso (siglo II d.C.) condenó la vivisección, pero aclaró que no era crueldad infligir sufrimiento a unos pocos cuando el beneficio era para muchos.

En la Edad Media el cristianismo prohibió la vivisección, pero no por esto dejó de realizarse. Leonardo Da Vinci (1452-1519), por ejemplo, hizo contribuciones a la anatomía comparada en perros y gatos, pero, aparentemente, predijo que algún día la experimentación en animales sería juzgada como un crimen(1). Andrés Vesalio (1514-1564) realizó demostraciones públicas de anatomía con vivisecciones en perros y cerdos(2). En aquel tiempo no se conocía la anestesia o analgesia adecuada y se justificó el sufrimiento causado con la necesidad de adquirir conocimientos y con la teoría de que los animales no tenían un alma racional. El cristianismo simplemente no consideraba que

1 En el resto del texto, al hablar de "animales" se hará referencia exclusivamente a los no humanos. tuvieran alma: Tomás de Aquino (siglo XIII) consideró que no había responsabilidad moral para con los animales. René Descartes (15961650) aseveró que las respuestas de éstos a estímulos dolorosos no eran más que reflejos, que eran autómatas y que no sentían ni pensaban de una manera racional y consciente. Sin embargo, contrastan algunos otros hechos históricos donde pareciera que, de alguna manera, se aceptaba que los animales eran responsables de sus actos; esto se puede ilustrar con el caso de una vaca condenada a morir en la horca por haber pateado a un hombre(3).

Tal vez uno de los primeros filósofos que señaló que los animales comparten la capacidad de sufrir y la conciencia con los humanos fue Schopenhauer (1788-1860)(4). Jeremy Bentham (1748-1832) también expresó dudas respecto de la opinión de Descartes, señalando: "la pregunta no es si pueden razonar o hablar, sino si pueden sufrir"(2).

Claude Bernard (1813-1878) publicó en 1865 , en medio de la gran influencia de la visión positivista de la ciencia y de los conflictos entre las opiniones filosóficas respecto del sufrimiento de los animales, su "Introducción el estudio de la medicina experimental" donde, al hablar sobre la vivisección, dice lo siguiente: "No se han podido descubrir las leyes de la materia bruta más que penetrando en los cuerpos y en las máquinas inertes; de la misma manera, no se podrán llegar a conocer las leyes y las propiedades de la materia viviente más que dislocando a los organismos para introducirse hasta su medio interior. Es pues absolutamente preciso, después de haber disecado en el cadáver, disecar en el vivo, para poner al descubierto $\mathrm{y}$ ver funcionar las partes interiores u ocultas del organismo. A esta especie de operaciones se les da el nombre de vivisecciones y sin este medio de investigación no hay fisiología, ni medicina científica posibles. Para saber cómo viven el hombre y los animales es indispensable 
ver morir a un gran número de ellos, porque los mecanismos de la vida no pueden ser revelados y probados sino por el conocimiento de los mecanismos de la muerte"(6). Esto hizo surgir una intensa polémica entre viviseccionistas y antiviviseccionistas. De hecho, la esposa y la hija del propio Bernard fueron fervientes militantes antiviviseccionistas.

En pleno siglo XIX surgió en 1860, en Gran Bretaña, la Real Sociedad para la Prevención de la Crueldad hacia los Animales, aprobándose en 1876 , por primera vez en el mundo, una Ley de Protección contra la Crueldad hacia los Animales. En 1882 se fundó en Suecia la Sociedad Nórdica contra Experimentos Dolorosos en Animales. No obstante, los animales siguieron siendo pieza clave en el estudio de varias enfermedades.

Iniciando el siglo XX Pavlov realizó varios experimentos en animales vivos; aunque, según algunos análisis, pudo haber estado adelantado a su tiempo respecto de las condiciones del trato ético con los animales(7). En este mismo siglo recrudeció la polémica sobre la experimentación en animales y nacieron nuevas controversias sobre la vivisección a raíz del surgimiento de la bioética.

\section{Ética de la relación entre humanos y animales}

Hasta bien entrado el siglo XX la ética tradicional ha sido antropocéntrica. Por un lado, hay un sentido epistemológico según el cual los humanos no pueden dejar de ser antropocéntricos, porque no pueden conocer a otros seres más que con la perspectiva netamente humana: algo así como unos anteojos epistémicos, ontológicamente irrenunciables. Por otro lado, las propuestas éticas que consideran a los animales no son epistémicas ni menos ontológicas: son éticas y contemplan la ampliación del círculo de seres a quienes se les considera un estatus moral.
Si bien la ética, en cuanto producto del intelecto humano, no puede dejar de ser antropocéntrica, puede en cambio ampliar el círculo de nuestras obligaciones morales hacia otros tipos de seres vivos. No se trata de introducir principios novedosos o extraños a la ética, sino de reinterpretar los existentes y/o ampliarlos hacia los animales.

Herrera Ibáñez cita tres formas de actitudes éticas hacia los animales: la aristotélica, la kantiana y la utilitarista(8). Para este trabajo se harán algunas observaciones partiendo de la postura kantiana clásica. Según Kant, un agente moral puede responder por sus actos y, guiado por la razón, toma la decisión libre y autónoma de actuar en un sentido o en otro. Un paciente moral es quien, siendo también libre, racional y autónomo, puede reclamar que otros cumplan su deber moral de respetarlo. Kant lo aclara en el famoso imperativo categórico, en su formulación práctica: "obra de tal modo que te relaciones con la humanidad, tanto en tu persona como en la de cualquier otro, siempre como un fin y nunca sólo como un medio"(9). El principio es claramente antropocéntrico, así como su noción de agente y paciente moral; sin embargo, no está exento de otras consideraciones.

Para Gracia Guillén, por ejemplo, es posible ampliar el imperativo categórico si se reinterpreta. Los animales constituyen un fin en su conjunto (a diferencia de los humanos, quienes lo serían considerados individualmente) y no solamente un medio, como tradicionalmente se les ha visto y se les ha tratado.

Tom Regan, deontólogo defensor de los derechos de los animales, afirma que los agentes morales son sujetos activos, artífices de la normativa ética y, como tales, tienen que responder por sus actos. Los pacientes morales, en cambio, son sujetos hacia los cuales se debe 
tener consideración moral, aunque ellos no tengan obligaciones morales para con los sujetos o no puedan reclamar respeto. En este grupo caben también ciertos grupos humanos, como los niños muy pequeños y los adultos con daño severo de sus funciones cognitivas (esquizofrenia, demencias, estado vegetativo permanente, entre otros). No obstante, no siempre queda tan claro que los animales pertenezcan a este grupo de los pacientes morales.

Hoy el consenso es que los animales son pacientes morales, es decir, tienen un estatus que debe de ser considerado en las acciones que tomen los agentes morales, es decir, los humanos(10).

\section{Ética y experimentación con animales}

Es una utopía insistir en cualquiera de los cursos extremos de la acción ética respecto de la experimentación con animales: aprobarla sin restricciones o negarla por completo. Desde la ética clásica aristotélica se enseña que la virtud no se encuentra en un extremo; antes bien, hay que buscarla en un punto intermedio. Los extremos respecto de la investigación con animales corresponderían seguramente a opciones viciosas y, por ello, sería imprudente recomendarlas. Como ha comentado Gilmore, el debate está cargado emocionalmente entre los que privilegian el avance del conocimiento médico y quienes apelan al respeto por la vida; en medio están quienes formulan (o al menos, deberían formular) guías éticas(11).

En 1954 la Universities Federation for Animal Welfare (UFAW) cambió su dirección de la crianza de animales de laboratorio hacia el tema controvertido de la experimentación animal. Designó a William M. S. Russell (zoólogo y psicólogo) y a Rex L. Burch (microbiólogo) para iniciar el estudio sistemático de técnicas experimentales desde una perspectiva ética. En 1959 ellos publicaron "The Principles of $\mathrm{Hu}$ - mane Experimental Techniques", de donde se extrae el ahora conocido "principio de las tres R": refinar, reducir y reemplazar. Adelantado para su tiempo, este texto es ahora ampliamente conocido y utilizado, y ningún científico responsable o publicación seria puede tratar el tema sin tenerlo como referencia(12).

Los métodos de refinamiento son los que alivian o minimizan el dolor, sufrimiento y estrés de los animales, de manera que se eleva su bienestar sin comprometer el resultado del experimento. Esto también conduce a investigaciones más seguras, ya que el dolor y el estrés generan cambios biológicos en el animal que pueden distorsionar el resultado. Incluye mejorar las condiciones de vida de los animales y su dieta, utilizar adecuadamente analgesia y anestesia, reducir el número de inyecciones y el volumen administrado, utilizar agujas del menor tamaño y calibre posible, pensar cuidadosamente en los parámetros a medir en el experimento, seleccionar los métodos menos invasivos y la especie adecuada, y utilizar el procedimiento eutanásico más humanizado $\mathrm{y}$ piadoso posible en caso de requerirlo.

Los métodos de reducción aspiran a conseguir niveles comparables de información con el uso de un menor número de animales, o a obtener más datos con el mismo número. La bioestadística aplicada convenientemente es muy valiosa para conseguir estos propósitos; hoy se puede afirmar que un mal diseño no lleva a un trabajo ético. Otra forma de reducción es la global, es decir, ¿cuánto se puede reducir la utilización de animales experimentales empleados en un laboratorio, región, país o en el mundo entero? En docencia, por ejemplo, cada vez se contempla más la aplicación de programas de simulación y la utilización de videos educativos, sobre todo cuando el educando no necesita imperiosamente adquirir habilidades en el manejo de animales experimentales. 
Los métodos de reemplazo permiten obtener resultados científicos sustituyendo a los vertebrados -comúnmente utilizados- por otros animales inferiores o sin la utilización de animales. En la actualidad no ha sido posible prescindir absolutamente de los animales de experimentación, pese a que existen muchos métodos: fisicoquímicos, modelos matemáticos y computacionales (interrelación cuantitativa estructura-actividad, diseño de fármacos con ayuda computacional, modelos matemáticos de procesos bioquímicos y fisiológicos, por ejemplo), uso de animales inferiores, uso de vertebrados en estadios tempranos del desarro1lo, cultivo de tejidos y metodologías ex vivo (o in vitro), estudios en seres humanos (cuando en ocasiones son los únicos que es posible utilizar como sujetos de investigación), utilización y extrapolación de la información existente, entre otros. Las ventajas de estas metodologías alternativas son de naturaleza científica y hasta económica, además de los evidentes beneficios humanitarios y éticos. Sin embargo, la existencia de algunas desventajas es lo que sigue poniendo un límite a su uso.

Para tópicos especiales en relación con la ética de la investigación utilizando animales se puede consultar el reporte que preparó recientemente The Nuffield Council on Bioethics(13).

\section{El método sincrético}

Gracia Guillén plantea en el sistema de referencia moral una premisa ontológica (el hombre es persona y, en cuanto tal, tiene dignidad y no precio) y otra moral (en tanto que personas, todos los hombres son iguales y merecen igual consideración y respeto). El esbozo moral (deontológico) lo sitúa desde el principialismo de Beauchamp y Childress, con un primer nivel (ética de mínimos) que involucra los principios de no maleficencia y justicia, y un segundo nivel (ética de máximos) que incluye los de beneficencia y autonomía.
La experiencia moral (teleológica) la enmarca previendo consecuencias objetivas o del nivel 1 y consecuencias subjetivas o del nivel 2. La verificación moral (justificación) consiste en contrastar la decisión con el sistema de referencia moral utilizado (contrastar el caso con la "regla" del esbozo moral) y comprobar si es posible justificar una "excepción" a la regla para el caso concreto analizado. Finalmente, tomar una decisión y someterla a varias pruebas, como el tiempo (si posteriormente se hubiera tomado la misma decisión), el marco jurídico local (si la decisión se encuentra dentro del marco legal del lugar donde se está trabajando) y la publicidad (si hubiera posibilidad de publicitar la decisión tomada) $(14,15)$.

Si bien esta construcción está centrada en el agente moral, se puede aplicar al trato con pacientes morales, como los animales. Por ejemplo, es posible extender el modelo pensando que la actividad de investigación tenga una estructura con un primer nivel en donde se parta de la no maleficencia, es decir, del "primero no dañar"(16). De esta forma, se puede incluir desde el primer nivel de una ética de mínimos para con los animales la consideración del principio de las tres R. El siguiente principio, la justicia, no podría aplicarse por igual si se habla de "animales", ya que habría que tener varias consideraciones de tipo gradualista respecto de su lugar en la escala evolutiva. $\mathrm{Si}$ se les considera a todos iguales se reducen las posibilidades de justificar la investigación en varias especies donde estaría plenamente validada con las consideraciones previas.

El siguiente nivel, la ética de máximos, incluye en humanos la autonomía y, a partir de ella, se determina lo que es bueno para cada quien. Toda vez que los animales no cuentan con autonomía tiene que haber agentes morales que determinen lo que para ellos sea bueno, en la medida de lo posible. La ética de máximos, en tanto no exigible, sólo es sugerida. 
Gracia ha sido un gran promotor y defensor de los comités de ética (asistenciales y de investigación) para promover la excelencia. Esta es una vía para conseguir la aplicación de la beneficencia con los animales, toda vez que carecen de autonomía: la creación de comités de ética que promuevan la excelencia en la investigación con esta clase de sujetos. El primer comité de ética de investigación animal fue creado en Suecia, en 1979, seguido por Estados Unidos, en 1984. Francia cuenta con regulaciones hasta de experimentación animal en el espacio. Sin embargo, en América Latina no fue sino hasta la década de los 90 cuando se crearon este tipo de comités(17).

\section{Conclusión}

¿Es éticamente aceptable realizar vivisección en animales? Considerando el modelo de la deliberación moral propuesto por Gracia Guillén, hay que analizar primero las opiniones de los implicados, identificar el problema ético, establecer los cursos de acción probables para tal problema, ponderar sus pros y los contras $\mathrm{y}$, de esta manera, elegir el mejor o, al menos, el menos lesivo para todas las partes. Todo ello tomando en cuenta el marco de la jerarquización de los principios en una ética de mínimos y otra de máximos.

Ante la posibilidad de realizar una vivisección en animales, hay que considerar opiniones de todos los implicados en el problema: investigadores, cuidadores de especies de laboratorio, bioeticistas, etólogos y psicólogos animales, biólogos, médicos veterinarios, entre otros. Sólo así se puede comprender la multidimensionalidad de este problema. Por otra parte, los animales son pacientes morales, es decir, tienen estatus moral: deben ser considerados, desde la perspectiva de la bioética, en la toma de decisiones por parte del agente moral.

Al analizar éticamente la vivisección animal, deberían enfrentarse al menos estas interrogantes: ¿cuáles son los argumentos que defienden la postura de realizar una vivisección animal? ¿Cuáles los beneficios potenciales y reales del hecho de realizarla? ¿Quién y cómo la realizaría? El hecho de viviseccionar, ¿fue revisado cuidadosamente por un Comité de Cuidado Animal? ¿Se está aplicando el principio de las tres R? ¿Se están empleando los mejores métodos de refinamiento para con los animales? ¿Existe un diseño adecuado desde el punto de vista de la bioestadística y de la metodología de la investigación científica? ¿Existe alguna forma de reemplazar la vivisección (en el caso de la docencia, con videos como ejemplo; en el caso de la investigación, con otro tipo de diseño que involucre otra especie)? ¿Se están respetando los documentos que muestran consensos y recomendaciones respecto del trato ético con los animales (Declaración de la Asociación Médica Mundial sobre el Uso de Animales en la Investigación Biomédica, adoptada por la $41^{\mathrm{a}}$ Asamblea Médica Mundial celebrada en Hong Kong, en 1989, la Guía del Consejo Internacional de Organizaciones de Ciencias Médicas para la Investigación Biomédica que Involucre Animales(18), las Guías para el Cuidado y Uso de los Animales de Laboratorio de los Institutos Nacionales de Salud de los Estados Unidos de $\operatorname{América(19),~etc.)?~}$

Como ha quedado asentado por Ushma Savla, "los científicos informados y bien entrenados tienen el privilegio, pero no el derecho automático, de utilizar animales como sujetos de investigación. No debe abusarse de este privilegio"(20). 


\section{Referencias}

1. Dolan K. Ethics, animals and science. London: Blackwell Science Ltd.; 1999.

2. Anaya Velázquez F, Garay Sevilla ME. Investigación en animales de laboratorio. En: Hernández Arriaga JL. Ética en la investigación biomédica. México, DF: Manual Moderno; 1999: 81-96.

3. Sechzer JA (ed.) The ethical dilemma of some classical animal experiments. The role of animals in biomedical research. New York: Annals Academic Sciences; 1983.

4. Schunemann de Aluja A. Consideraciones éticas en la experimentación científica con animales y Norma Oficial Mexicana NOM062-ZOO-1999: especificaciones técnicas para la producción, cuidado y uso de los animales de laboratorio. En: Velasco Suárez M, Cano Valle F, Torres Mejía J (eds.) Memoria del V Congreso Nacional, Latinoamericano y del Caribe de Bioética. México, DF: Comisión Nacional de Bioética y Academia Nacional Mexicana de Bioética; 2002: 207-218.

5. Pratt D. Alternatives to pain in experiments on animals. New York: Argus Archives; 1980.

6. Bernard C. Introducción al estudio de la medicina experimental. Puebla: Universidad Autónoma de Puebla; 1987.

7. Kopaladze RA. Ivan P. Pavlov's view on vivisection. Integr Physiol Behav Sci 2000; 35(4): 266-271.

8. Herrera Ibáñez A. Tres actitudes éticas hacia los animales. En: Cano Valle F, Torres Mejía J (eds.) Memoria del VI Congreso Nacional, Latinoamericano y del Caribe de Bioética. México, DF: Comisión Nacional de Bioética; 2002: 197-203.

9. Kant I. Fundamentación de la metafísica de las costumbres. Madrid: Espasa-Calpe; 1995: 104.

10. Álvarez Díaz JA. El estatus moral de los animales como problema bioético. En: Álvarez Díaz JA, Campbell Manjarrez U. (Comps.) La bioética en la frontera. Ciudad Juárez: Universidad Autónoma de Ciudad Juárez; 2006: 70-84.

11. Gilmore A. The use of animals in research. Can Med Assoc J 1985;132(5):564-8, 570-1.

12. Álvarez Díaz JA, Cardozo C. Ética de la investigación biomédica que usa y cuida animales experimentales. En: Lolas F, Quezada A, Rodríguez E. (eds.) Investigación en salud. Dimensión ética. Santiago de Chile: CIEB, Universidad de Chile; 2006: 239-248.

13. The Nuffield Council on Bioethics. The ethics of research involving animals. London: 2005. [Sitio en Internet] Disponible en http://www.nuffieldbioethics.org/go/ourwork/animalresearch/publication_178. html Último acceso en marzo de 2007.

14. Gracia D. Fundamentos de bioética. Madrid: Eudema; 1989.

15. Gracia D. Procedimientos de decisión en ética clínica. Madrid: Eudema; 1993.

16. Nelson RJ, Mandrell TD. Enrichment and nonhuman primates: "first, do no harm". ILAR J 2005;46(2): 171-177.

17. Brandao Schnaider T, de Souza C. Aspectos eticos da experimentação animal. Rev Bras Anestesiol 2003;53(2):278-285. 
La controversia sobre la vivisección - J. Álvarez-Díaz

18. Council for the International Organization of Medical Sciences (CIOMS). Biomedical research involving animals. International Guiding Principles. Geneva: CIOMS; 1993.

19. National Institutes of Health. Guide for the care and use of laboratory animals. Maryland: Division of research resources; 1994.

20. Savla U. Responsible conduct in animal research. J Clin Invest 2003; 112(10): 1456.

Recibido el 18 de octubre de 2006.

Aceptado el 13 de febrero de 2007. 